(2) OPEN ACCESS

\title{
Endoscopic placement of covered versus uncovered self-expandable metal stents for palliation of malignant gastric outlet obstruction
}

\author{
Kentaro Yamao, ${ }^{1}$ Masayuki Kitano (D) , ${ }^{1,2}$ Yasutaka Chiba, ${ }^{3}$ Takeshi Ogura (D) , \\ Takaaki Eguchi, ${ }^{5}$ Ichiro Moriyama, ${ }^{6}$ Yukitaka Yamashita, ${ }^{7}$ Hironari Kato, ${ }^{8}$ \\ Takahisa Kayahara, ${ }^{9}$ Noriyuki Hoki, ${ }^{10}$ Yoshinobu Okabe, ${ }^{11}$ Hideyuki Shiomi, ${ }^{12}$ \\ Yoshitaka Nakai, ${ }^{13}$ Yoshinori Kushiyama, ${ }^{14}$ Yoshifumi Fujimoto, ${ }^{15}$ Shiro Hayashi, ${ }^{16}$ \\ Shigeki Bamba (D) , ${ }^{17}$ Yasushi Kudo, ${ }^{18}$ Nobuaki Azemoto, ${ }^{19}$ Toshiharu Ueki, ${ }^{20}$ \\ Norimitsu Uza, ${ }^{21}$ Masanori Asada, ${ }^{22}$ Kazuya Matsumoto, ${ }^{23}$ Hiroko Nebiki, ${ }^{24}$ \\ Hiroshi Takihara, ${ }^{25}$ Chisio Noguchi, ${ }^{26}$ Hideki Kamada ${ }^{27}$ Kojiro Nakase $^{28}$ \\ Daisuke Goto, ${ }^{29,30}$ Tsuyoshi Sanuki, $^{31}$ Tetsuya Koga, ${ }^{32}$ Shinichi Hashimoto, ${ }^{33}$ \\ Hidefumi Nishikiori, ${ }^{34}$ Masahiro Serikawa, ${ }^{35}$ Keiji Hanada, ${ }^{36}$ Ken Hirao, ${ }^{37}$ \\ Masaya Ohana, ${ }^{38}$ Imakiire Kazuyuki, ${ }^{39}$ Takao Kato, $^{40}$ Motoyuki Yoshida, ${ }^{41}$ \\ Hirofumi Kawamoto ${ }^{42}$
}

- Additional material is published online only. To view, please visit the journal online (http://dx.doi.org/10.1136 gutjnl-2020-320775).

For numbered affiliations see end of article.

Correspondence to Professor Masayuki Kitano, Second Department of Internal Medicine, Wakayama Medical University, Wakayama, Wakayama 641-0012, Japan; kitano@wakayama-med.ac.jp

Received 28 January 2020 Revised 27 October 2020 Accepted 29 October 2020 Published Online First 22 November 2020
Check for updates

(c) Author(s) (or their employer(s)) 2021. Re-use permitted under CC BY-NC. No commercial re-use. See rights and permissions. Published by BMJ.

To cite: Yamao K, Kitano M, Chiba Y, et al. Gut

2021:70:1244-1252.

\section{ABSTRACT}

Objective Stenting is an established endoscopic therapy for malignant gastric outlet obstruction (mGOO). The choice of stent (covered vs uncovered) has been examined in prior randomised studies without clear results.

Design In a multicentre randomised prospective study, we compared covered (CSEMS) with uncovered self-expandable metal stents (UCSEMS) in patients with mGO0; main outcomes were stent dysfunction and patient survival, with subgroup analyses of patients with extrinsic and intrinsic tumours.

Results Overall survival was poor with no difference between groups (probability at 3 months $49.7 \%$ for covered vs $48.4 \%$ for uncovered stents; log-rank for overall survival $p=0.26$ ). Within that setting of short survival, the proportion of stent dysfunction was significantly higher for uncovered stents ( $35.2 \%$ vs $23.4 \%, p=0.01$ ) with significantly shorter time to stent dysfunction. This was mainly relevant for patients with extrinsic tumours (stent dysfunction rates for uncovered stents $35.6 \%$ vs $17.5 \%, p<0.01$ ). Subgrouping was also relevant with respect to tumour ingrowth (lower with covered stents for intrinsic tumours; $1.6 \%$ vs $27.7 \%$, $p<0.01$ ) and stent migration (higher with covered stents for extrinsic tumours: $15.3 \%$ vs $2.5 \%, p<0.01$ ).

Conclusions Due to poor patient survival, minor differences between covered and uncovered stents may be less relevant even if statistically significant; however, subgroup analysis would suggest to use covered stents for intrinsic and uncovered stents for extrinsic malignancies.

\section{INTRODUCTION}

Malignant gastric outlet obstruction (mGOO) can occur late in patients with upper digestive and pancreatobiliary cancer. Endoscopic gastroduodenal stent (GDS) deployment or surgical gastrojejunostomy plays an important role in maintaining a long oral intake duration. Endoscopic GDS deployment for mGOO has recently become more common, effective, safer and less expensive than gastrojejunostomy. ${ }^{1-4}$ While GDS deployment has excellent technical and clinical success, stent dysfunction (SD) and other adverse events may occur depending on whether an uncovered selfexpandable metal stent (UCSEMS) or covered selfexpandable metal stent (CSEMS) is used. The main cause of UCSEMS dysfunction is stent ingrowth, and the CSEMS was developed to prevent stent ingrowth. The CSEMS is associated with a lower occlusion risk but higher migration risk according to a systematic review and meta-analysis. ${ }^{5}$ The cause of CSEMS migration is the presence of the stent cover. Additionally, the mechanism of stent migration may differ depending on whether the main tumour is extrinsic or intrinsic. Pancreatic and gallbladder cancers compress the digestive tract extrinsically. Conversely, gastric and duodenal cancers arising from the epithelial cells of the digestive tract wall cause stenosis intrinsically.

A CSEMS with a large uncovered flare at the proximal end was recently developed to prevent CSEMS migration. In this multicentre randomised trial, we compared this novel CSEMS with a large uncovered flare versus the UCSEMS in terms of SD. Comparisons were made among all patients with $\mathrm{mGOO}$ and those with extrinsic and intrinsic tumours separately.

\section{MATERIALS AND METHODS}

\section{Patients}

Consecutive patients who had symptomatic mGOO with unresectable malignant tumours requiring placement of a SEMS were enrolled. The inclusion 
Significance of this study

What is already known on this subject?

- The main cause of uncovered self-expandable metal stent (UCSEMS) dysfunction is stent ingrowth, while the main cause of covered self-expandable metal stent (CSEMS) dysfunction is stent migration.

- Many studies have assessed the clinical results between patients with a CSEMS and UCSEMS, but the final choice between the two types of self-expandable metal stent (SEMS) remains unclear.

\section{What are the new findings?}

- No difference in the proportion of stent ingrowth between the two groups was found for patients with extrinsic tumours; however, this stent ingrowth was significantly lower among patients with intrinsic tumours in the CSEMS group than the UCSEMS group.

- The proportion of stent migration among patients with extrinsic tumours was significantly higher in the CSEMS group than the UCSEMS group.

- The main message, however, is that the life span of patients with this disease is rather short; therefore, subgrouping may help to stratify these patients.

\section{How might it impact on clinical practice in the foreseeable} future?

- A UCSEMS may be used among patients with malignant gastric outlet obstruction, particularly those with extrinsic tumours, because a lower proportion of stent dysfunction is expected. criteria were as follows: (1) obstruction-related symptoms, including vomiting and ingestion of only liquid or no oral intake, (2) performance status of $0-2$ and (3) unresectable malignant obstruction confirmed by preceding endoscopy and histology. The exclusion criteria were as follows: (1) another obstruction at the anal side of the intestine confirmed by preceding enterography, (2) disturbance of motility due to peritonitis carcinomatosa, (3) severe ascites extending to both the abdominal and pelvic cavities, (4) previous treatment of GOO, (5) inability to perform endoscopy because of a severe general condition and (6) refusal to participate in the study.

\section{Study design}

This multicentre, open-label study was conducted with prospective randomisation and compared clinical outcomes and adverse events between CSEMS and UCSEMS. The list of participating institutions is shown in online supplemental table 1 . The study was performed according to the guidelines described in the Declaration of Helsinki for biomedical research involving human subjects (Clinical trial registration number: University Hospital Medical Information Network 000013653), and all patients provided written informed consent.

The patients were randomised for CSEMS or UCSEMS deployment in a 1:1 fashion. Randomisation was stratified by the cause of mGOO (intrinsic or extrinsic) and the oral site of the stenosis (stomach, bulb, second portion of the duodenum, or third and fourth portions of the duodenum). Randomisation was performed using a web-based system designed by an independent research organisation (Tofield Company Limited, Tokyo, Japan).

Figure 1 Covered and uncovered self-expandable metallic stent (SEMS) used in this study. (A) The covered SEMS has an antimigration system at both ends (proximal uncovered flare and distal uncovered area). (B) The uncovered SEMS is straight and contains no membrane. 


\section{Characteristics of SEMS}

The CSEMS used in this study was the flared-ComVi stent (Taewoong Medical, Goyang, Korea) (figure 1A), which had a covered polytetrafluoroethylene membrane. Its length was $60,80,100$ or $120 \mathrm{~mm}$ depending on the stenosis length. All CSEMSs were $20 \mathrm{~mm}$ in diameter and had an antimigration system at both ends; the proximal uncovered flare was $15 \mathrm{~mm}$ in length and $25 \mathrm{~mm}$ in diameter, and the distal uncovered area was $15 \mathrm{~mm}$ in length and $20 \mathrm{~mm}$ in diameter. The proximal flare was expected to prevent stent migration by fixation to the gastroduodenal wall. The UCSEMS used in this study was the Niti-S pyloric/duodenal D-type stent (Taewoong Medical) (figure 1B), which was made of nitinol wire. Its length was 60, 80, 100 or $120 \mathrm{~mm}$. All USEMSs were equipped with no flares and were 22 $\mathrm{mm}$ in diameter.

\section{Stent placement}

All SEMSs were deployed under endoscopic and fluoroscopic guidance. Patients were sedated with intravenous midazolam and/or propofol. For GDS deployment, a therapeutic endoscope (GIF 1T-240, 2T-240 or TJF 260; Olympus Medical Systems, Tokyo, Japan) was used. An endoscopic retrograde cholangiopancreatography catheter with a biliary guidewire was used. Thus, the endoscope was first allowed to approach the gastric or duodenal stenosis site, after which a guidewire equipped with a catheter was passed through as far away from the stenosis site as possible. The distal or proximal lumen of the stenosis site was captured by fluoroscopy, and after confirming the position and length of the stenosis site, we determined the appropriate length and position of the stent. Thereafter, while considering the shortening of the stent after extension, the GDS was placed under endoscopic and fluoroscopic guidance.

\section{Follow-up}

Patients without immediate adverse events were allowed to start clear fluid intake 1-5 days after GDS placement. An abdominal radiograph was obtained 1-3 days after the intervention to check the stent expansion and location. If the patient showed no GOO symptoms, no stent dislocation and adequate stent expansion after receiving clear fluids, a semisolid diet was started. The severity of GOO was assessed before and after stent placement using an adaptation of the GOO scoring system (GOOSS), ${ }^{6}$ in which swallowing ability is divided into four categories: 0 , no oral intake; 1, liquids only; 2, soft solids; and 3, low residue or full diet. SD was defined as appetite loss, nausea and/or vomiting with a GOOSS score of $<2$ and confirmation of obstruction by any imaging technique (endoscopy, fluoroscopy, CT and/or ultrasonography). The causes of SD included stent ingrowth, overgrowth, stent migration, kinking, food impaction, stent fracture and unsatisfactory stent expansion. These causes of SD were mainly determined endoscopically and/or fluoroscopically but were assessed by CT or ultrasonography in some patients with a worse general condition. In such cases, SD of which the cause was not detected regardless of oral-side gastroduodenal stent expansion was treated as an unknown cause. Blood examination was performed on days 1 and 7 after stenting. In principle, all patients were followed up monthly by physical examination and standard blood tests until death. For patients who were transferred to another hospital, the date of death was determined by telephone contact.

\section{Outcome measurements and definitions}

The primary aim was to compare the time to SD between the CSEMS and UCSEMS groups, for which the analysis set was intention-to-treat (ITT). The secondary aims were to evaluate the proportion of SD throughout the observation period, the probability of no SD at 1, 2 and 3 months, and the survival probability; determine the proportions of technical success, clinical success and adverse events; and identify factors that predict SD at 2 months. In subgroup analyses, time to SD; proportion of SD throughout the observation period; and probability of no SD at 1,2 and 3 months were compared among patients with each tumour type (extrinsic vs intrinsic tumours) and stenotic site (duodenum vs stomach) separately. Time to SD was measured from the day of stent placement to SD. Patients who died without SD were censored for time to SD. Patients who underwent surgery because of improvement of the primary disease by chemotherapy and/or radiation were censored for time to SD on the day of surgery. Survival time was measured from the day of stent placement to patient death. Technical success was defined as adequate SEMS placement across the stenosis as confirmed by endoscopy and fluoroscopy. Clinical success was defined as a GOOSS score of $\geq 2$ and relief of GOO symptoms within 1 week after stent insertion. Causes of GOO were divided into two categories: extrinsic and intrinsic tumours. An extrinsic tumour was defined as a tumour that compressed and/or invaded the digestive tract from the outside. The representative extrinsic tumours were pancreatic, gallbladder and similar cancers. An intrinsic tumour was defined as a tumour that obstructed the digestive tract directly from the inside. The representative intrinsic tumours were gastric, duodenal and similar cancers. The stenotic site was defined as the oral side of the stenosis.

We also explored prognostic factors that were independently associated with SD at 2 months. The following factors were evaluated for their ability to predict SD: sex, age, tumour type (extrinsic), stenotic site (duodenum), performance status, use of chemotherapy after SEMS placement and type of SEMS (CSEMS).

\section{Sample size and statistical analysis}

For sample size estimation, we retrospectively assessed stent effectiveness in all participating hospitals before this study; the probability of no SD with a UCSEMS at 2 months was $86.6 \%$ (355 out 410 cases), by which we assumed it to be $90 \%$. Under the assumption of a HR of 0.5 , the required number of patients was calculated as 175 in each group based on the log-rank test, with a significance level of 0.05 (two-sided) and power of 0.8 . Accounting for withdrawal of a few patients, the planned sample size was set at 190 patients in each group.

Continuous variables are expressed as mean \pm SD and were compared using the Wilcoxon signed-rank test. Categorical data are expressed as n (\%) and were compared using Fisher's exact test. The cumulative time to SD and survival time were evaluated using Kaplan-Meier analysis. A logistic regression analysis was applied to explore prognostic factors that were independently associated with SD at 2 months. ORs and 95\% CIs were reported. All statistical analyses were performed using SPSS Statistics V.19 (IBM). A p value of $<0.05$ was considered statistically significant.

\section{RESULTS}

\section{Recruitment and participant flow}

Recruitment was performed from April 2014 to March 2017, and the final follow-up was completed in September 2017. During the study period, 563 patients were initially considered for the study (figure 2). In total, 197 patients met the exclusion criteria. Ultimately, 366 patients were randomised, and 


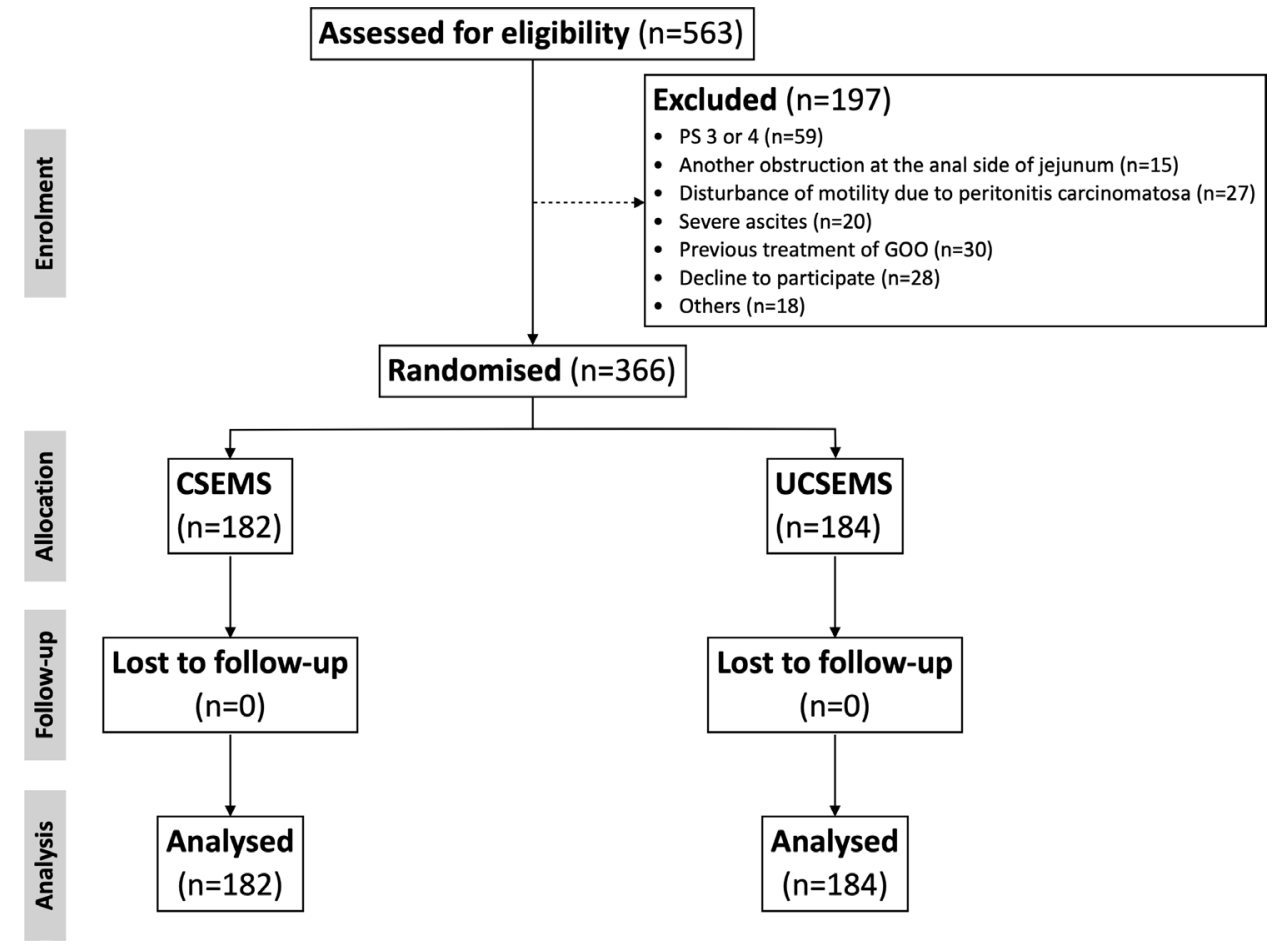

Figure 2 CONSORT (Consolidated Standards of Reporting Trials) flow diagram of patient enrolment. CSEMS, covered self-expandable metal stent; GOO, gastric outlet obstruction; UCSEMS, uncovered self-expandable metal stent.

182 patients from the CSEMS group and 184 patients from the UCSEMS group were included in the analysis, in which the analysis set was ITT.

\section{Patient characteristics in overall CSEMS and UCSEMS groups}

The primary disease in the CSEMS and UCSEMS groups was pancreatic cancer in $48.9 \%$ and $48.9 \%$ of patients, gastric cancer in $31.3 \%$ and $33.2 \%$, gallbladder cancer in $5.5 \%$ and $6.5 \%$, and others in $14.3 \%$ and $11.4 \%$, respectively. The tumour type in each group was an intrinsic tumour in $35.2 \%$ and $35.3 \%$ of patients and an extrinsic tumour in $64.8 \%$ and $64.7 \%$, respectively. There were no statistically significant differences in the other patient characteristics between the two groups (table 1).

\section{Patient survival between CSEMS and UCSEMS groups}

No difference in overall survival was found between the two groups $(\log$-rank $\mathrm{p}=0.26)$. The probability at 1,2 and 3 months was $85.1 \%, 66.6 \%$ and $49.7 \%$ in the CSEMS group and $85.8 \%$, $64.5 \%$ and $48.4 \%$ in the UCSEMS group, respectively (figure 3 ).

\section{Technical success, clinical success and adverse events between CSEMS and UCSEMS groups}

Technical and clinical success were achieved in high proportions of patients, and no differences were found between the two groups ( $p=1.00$ and 0.69 , respectively). Additionally, no differences were found in the incidence of overall or each adverse event (table 2).

\section{Stent dysfunction in overall patients between CSEMS and UCSEMS groups}

Among the overall patients, the CSEMS group showed a significantly higher proportion of overall SD (35.2\% vs $23.4 \%$, $\mathrm{p}=0.01)$, stent overgrowth $(\mathrm{p}=0.04)$ and stent migration $(p<0.01)$ than the UCSEMS group. However, the opposite result was obtained for stent ingrowth $(p<0.01)$ (table 2$)$. The time to SD was significantly shorter in the CSEMS than the UCSEMS group $(\log$-rank $\mathrm{p}<0.01)$. Its probability at 1,2 and 3 months was $83.3 \%, 75.6 \%$ and $69.1 \%$ in the CSEMS group and $92.2 \%, 88.3 \%$ and $83.7 \%$ in the UCSEMS group, respectively (figure 4A).

\section{Subgroup analysis of stent dysfunction using tumour type}

Among patients with extrinsic tumours, the CSEMS group showed a significantly higher incidence of overall SD $(35.6 \%$ vs $17.5 \%, p<0.01)$ and stent migration $(p<0.01)$ than the UCSEMS group, but no differences in the proportions of stent ingrowth $(p=0.70)$ and stent overgrowth $(p=0.36)$ were found between the CSEMS and UCSEMS groups (table 3 ). The time to SD was significantly shorter in the CSEMS group than the UCSEMS group (log-rank p<0.01).

Among patients with intrinsic tumours, the CSEMS group showed a significantly lower incidence of stent ingrowth than the UCSEMS group $(\mathrm{p}<0.01)$, but no differences in the proportion of overall SD $(34.4 \%$ vs $33.8 \%, p=0.98)$, stent overgrowth $(p=0.12)$ or stent migration $(p=0.35)$ were found between the CSEMS and UCSEMS groups (table 3). Additionally, no differences in the time to SD were found between the two groups $(\log$-rank $\mathrm{p}=0.14)$.

\section{Subgroup analysis of stent dysfunction using stenotic site}

Among patients with duodenal stenosis, the proportion of SD was significantly higher $(p=0.02)$ (online supplemental table 2) and the time to SD was significantly shorter in the CSEMS group than the UCSEMS group (log-rank $\mathrm{p}=0.03$ ) (online supplemental figure $1 \mathrm{~A})$. 


\begin{tabular}{|c|c|c|c|}
\hline & CSEMS & UCSEMS & \\
\hline & $\mathrm{n}=182$ & $\mathrm{n}=184$ & P value \\
\hline \multicolumn{4}{|l|}{ Sex } \\
\hline Male & $98(53.8)$ & $107(58.2)$ & 0.41 \\
\hline Female & $84(46.2)$ & $77(41.8)$ & \\
\hline Age, years & $73.5(35-97)$ & $72(43-96)$ & 0.99 \\
\hline Tumour diagnosis & & & 0.93 \\
\hline Pancreatic cancer & $89(48.9)$ & $90(48.9)$ & \\
\hline Gastric cancer & $57(31.3)$ & $61(33.2)$ & \\
\hline Gallbladder cancer & $10(5.5)$ & $12(6.5)$ & \\
\hline Other & $26(14.3)$ & $21(11.4)$ & \\
\hline \multicolumn{4}{|l|}{ Tumour type } \\
\hline Intrinsic & $64(35.2)$ & $65(35.3)$ & 0.97 \\
\hline Extrinsic & $118(64.8)$ & $119(64.7)$ & \\
\hline \multicolumn{4}{|l|}{ Stenotic site } \\
\hline Stomach & $63(34.6)$ & $59(32.1)$ & 0.60 \\
\hline Duodenum & $119(65.4)$ & $125(67.9)$ & \\
\hline D1 (bulb) & $50(27.5)$ & $47(25.5)$ & \\
\hline D2 (second portion) & $31(17.0)$ & $35(19.0)$ & \\
\hline D3 (third portion) & $35(19.2)$ & $38(20.7)$ & \\
\hline D4 (fourth portion) & $3(1.6)$ & $5(2.7)$ & \\
\hline \multicolumn{4}{|l|}{ GOOSS score before SEMS placement } \\
\hline 0 & $82(45.1)$ & $95(51.6)$ & 0.21 \\
\hline 1 & $100(54.9)$ & $89(48.4)$ & \\
\hline \multicolumn{4}{|l|}{ Performance status } \\
\hline 0 & $49(26.9)$ & $62(33.6)$ & 0.22 \\
\hline 1 & $80(44.0)$ & $65(35.3)$ & \\
\hline 2 & $53(29.1)$ & $57(30.9)$ & \\
\hline \multicolumn{4}{|l|}{ Stage } \\
\hline IV & $167(91.8)$ & $164(89.1)$ & 0.72 \\
\hline III & $12(6.6)$ & $16(8.7)$ & \\
\hline Other & $3(1.6)$ & $4(2.2)$ & \\
\hline Chemotherapy after SEMS placement & $66(36.3)$ & $70(38.0)$ & 0.72 \\
\hline
\end{tabular}

Data are presented as $n(\%)$ or median (range).

CSEMS, covered self-expandable metal stent; GOOSS, gastric outlet obstruction scoring system; SEMS, self-expandable metal stent; UCSEMS, uncovered self-expandable metal stent.

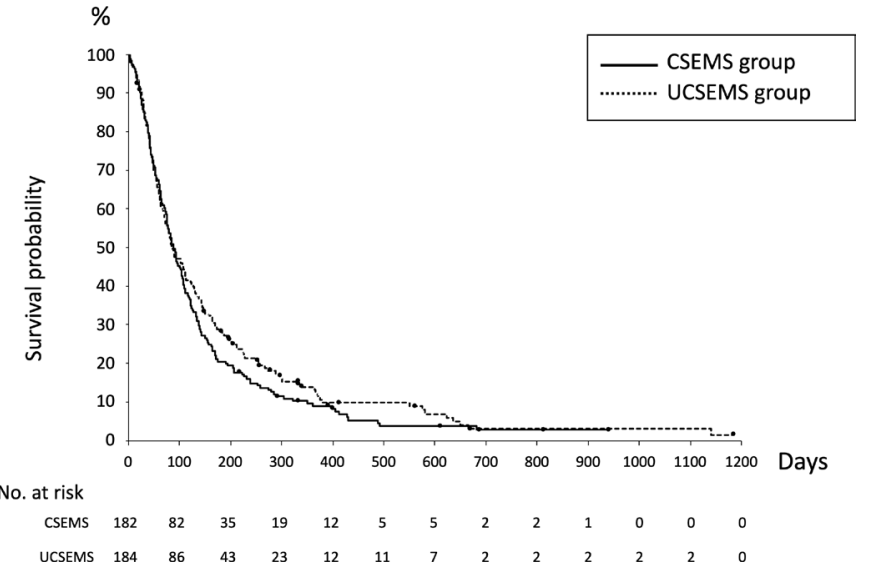

Figure 3 Kaplan-Meier curves for patient survival. The survival probability at 1, 2 and 3 months was $85.1 \%, 66.6 \%$ and $49.7 \%$ in the covered self-expandable metal stent (CSEMS) group and $85.8 \%, 64.5 \%$ and $48.4 \%$ in the uncovered self-expandable metal stent (UCSEMS) group, respectively (log-rank $\mathrm{p}=0.26)$.
Table 2 Clinical results among the overall patients between the CSEMS and UCSEMS groups

\begin{tabular}{lccc}
\hline & $\begin{array}{l}\text { CSEMS } \\
\mathrm{n}=182\end{array}$ & $\begin{array}{l}\text { UCSEMS } \\
\mathrm{n}=184\end{array}$ & P value \\
\hline Technical success & $182(100)$ & $184(100)$ & 1.00 \\
\hline Clinical success & $164(90.1)$ & $168(91.3)$ & 0.69 \\
Overall adverse events & $39(21.4)$ & $37(20.1)$ & 0.76 \\
\hline Jaundice and/or cholangitis & $23(12.6)$ & $18(9.8)$ & 0.39 \\
\hline Bleeding & $3(1.6)$ & $6(3.3)$ & 0.51 \\
Perforation & $3(1.6)$ & $3(1.6)$ & 0.69 \\
\hline Pancreatitis & $2(1.1)$ & $2(1.1)$ & 0.62 \\
\hline Other & $8(4.4)$ & $8(4.3)$ & 0.82 \\
\hline Overall stent dysfunction & $64(35.2)$ & $43(23.4)$ & 0.01 \\
\hline Stent ingrowth & $6(3.3)$ & $23(12.5)$ & $<0.01$ \\
Stent overgrowth & $12(6.6)$ & $4(2.2)$ & 0.04 \\
\hline Stent migration & $22(12.1)$ & $4(2.2)$ & $<0.01$ \\
\hline Other & $24(13.2)$ & $12(6.5)$ & 0.03 \\
\hline
\end{tabular}

Data are presented as $n(\%)$

CSEMS, covered self-expandable metal stent; NA, not applicable; UCSEMS, uncovered self-expandable metal stent.

Among patients with gastric stenosis, no difference in the proportion of SD was found $(p=0.30)$ (online supplemental table 2); however, the time to SD was significantly shorter in the CSEMS group than the UCSEMS group (log-rank $\mathrm{p}=0.01)$ (online supplemental figure 1B).

In the analysis of the stenotic site, the following outcomes were similar in comparison of the tumour type: the proportion of overall SD and each type of SD (except stent migration in the gastric stenosis group) (online supplemental table 2). However, regarding stent migration, the outcome of the analysis of the stenotic site was different in comparison of the tumour type. The proportion of stent migration among patients with
(A)

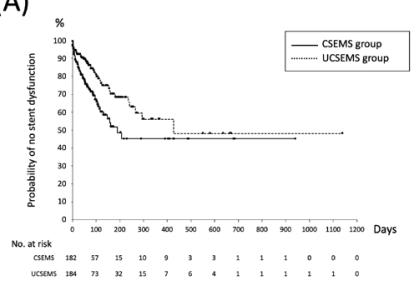

(C)

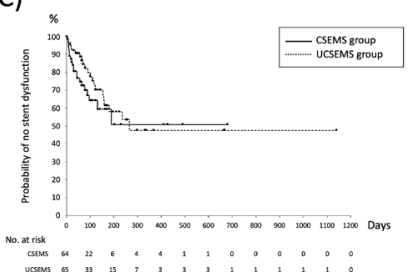

(B)

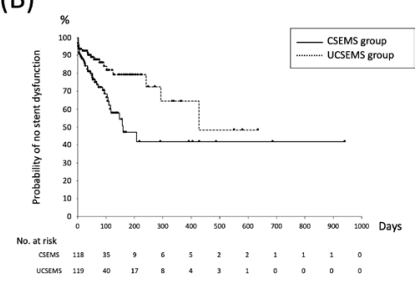

Figure 4 Kaplan-Meier curves for stent dysfunction (SD). (A) KaplanMeier curves for SD among overall patients. The probability of no SD at 1,2 and 3 months was $83.3 \%, 75.6 \%$ and $69.1 \%$ in the CSEMS group and $92.2 \%, 88.3 \%$ and $83.7 \%$ in the UCSEMS group, respectively (log-rank $\mathrm{p}<0.01)$. (B) Kaplan-Meier curves for SD among patients with extrinsic tumours. The probability of no SD at 1, 2 and 3 months was $84.1 \%, 76.2 \%$ and $70.4 \%$ in the CSEMS group and $92.3 \%, 88.2 \%$ and $85.9 \%$ in the UCSEMS group, respectively (log-rank $\mathrm{p}<0.01$ ). (C) Kaplan-Meier curves for SD among patients with intrinsic tumours. The probability of no SD at 1,2 and 3 months was $82.1 \%, 74.5 \%$ and $67.1 \%$ in the CSEMS group and $92.2 \%, 88.6 \%$ and $81.1 \%$ in the UCSEMS group, respectively (log-rank $\mathrm{p}=0.14)$. 
Table 3 Stent dysfunction by comparison of tumour type (extrinsic vs intrinsic tumour) between the CSEMS and UCSEMS groups

\begin{tabular}{lccc}
\hline & CSEMS & UCSEMS & P value \\
\hline Extrinsic tumour & $\mathrm{n}=118$ & $\mathrm{n}=119$ & \\
Overall stent dysfunction & $42(35.6)$ & $21(17.5)$ & $<0.01$ \\
\hline Stent ingrowth & $5(4.2)$ & $5(4.2)$ & 0.70 \\
\hline Stent overgrowth & $8(6.8)$ & $4(3.3)$ & 0.36 \\
\hline Stent migration & $18(15.3)$ & $3(2.5)$ & $<0.01$ \\
\hline Other & $11(9.3)$ & $9(7.6)$ & 0.63 \\
Intrinsic tumour & $\mathrm{n}=64$ & $\mathrm{n}=65$ & \\
Overall stent dysfunction & $22(34.4)$ & $22(33.8)$ & 0.98 \\
\hline Stent ingrowth & $1(1.6)$ & $18(27.7)$ & $<0.01$ \\
\hline Stent overgrowth & $4(6.3)$ & $0(0.0)$ & 0.12 \\
\hline Stent migration & $4(6.3)$ & $1(1.5)$ & 0.35 \\
Other & $13(20.3)$ & $3(4.6)$ & $<0.01$ \\
\hline
\end{tabular}

Data are presented as $n(\%)$

CSEMS, covered self-expandable metal stent; UCSEMS, uncovered self-expandable metal stent.

gastric stenosis was significantly higher in the CSEMS than the UCSEMS group $(p=0.02)$ (online supplemental table 2 ). In the CSEMS group among patients with gastric stenosis, 10 patients had pancreatic cancer, and 4 of these patients developed stent migration.

\section{Predictive factors for stent dysfunction}

A logistic regression analysis was performed to explore the factors affecting SD at 2 months. The results revealed that CSEMS deployment (OR 2.41; $\mathrm{p}<0.01$ ) and age (OR 0.98; $\mathrm{p}=0.04$ ) were the factors that affected SD at 2 months (table 4 ).

\section{DISCUSSION}

This is the largest multicentre prospective study to compare a CSEMS with a large uncovered flare versus a UCSEMS among patients with mGOO. We had hypothesised that this novel CSEMS was unlikely to be associated with stent migration because of the presence of the large uncovered flare, and we had expected this novel CSEMS to contribute to prevention of SD. However, the proportion of SD was significantly higher and the time to SD among the overall patients was significantly shorter in the CSEMS than the UCSEMS group. Nevertheless, this is the first study to compare the clinical results among patients with extrinsic and intrinsic tumours separately. Among patients with extrinsic tumours, the proportion of SD was significantly higher and the time to SD was significantly shorter in the CESMS than UCSEMS group. In contrast, no differences in these parameter were found for the patients with intrinsic tumours between the two groups.

Table 4 Multivariable analysis of factors associated with stent dysfunction at 2 months

\begin{tabular}{lccc}
\hline & OR & $95 \% \mathrm{Cl}$ & P value \\
\hline Sex, male & 0.87 & 0.58 to 1.29 & 0.48 \\
\hline Age, years & 0.98 & 0.95 to 1.00 & 0.04 \\
Tumour type, extrinsic & 1.35 & 0.60 to 3.04 & 0.47 \\
Stenotic site, duodenum & 0.54 & 0.25 to 1.20 & 0.13 \\
Performance status 2 & 1.03 & 0.55 to 1.92 & 0.92 \\
Chemotherapy after SEMS placement & 0.62 & 0.33 to 1.17 & 0.14 \\
CSEMS & 2.41 & 1.34 to 4.33 & $<0.01$ \\
\hline
\end{tabular}

CSEMS, covered self-expandable metal stent; SEMS, self-expandable metal stent.
Many studies have assessed the clinical results between patients with a CSEMS and UCSEMS after placement of a GDS, ${ }^{1-5}$ 7-25 including four randomised controlled trials (RCTs). ${ }^{8} 12 \quad 1316$ One systematic review and meta-analysis showed no significant difference in the proportion of SD between the two groups but revealed a significantly lower incidence of stent occlusion, including stent ingrowth and a higher incidence of stent migration when using a CSEMS than UCSEMS. ${ }^{5}$ Another systematic review and meta-analysis showed that a CSEMS can reduce the risk of restenosis, whereas a UCSEMS is effective in decreasing stent migration. ${ }^{25}$

In previous RCTs that compared the CSEMS and UCSEMS, the incidence of stent ingrowth was $0.0 \%-3.4 \%$ in the CSEMS group and 16.1\%-44.4\% in the UCSEMS group. ${ }^{8} 121316$ In most studies, the incidence of stent ingrowth was significantly lower in the CSEMS group than the UCSEMS group. ${ }^{8} 1213$ In the present study, the incidence of stent ingrowth in the CSEMS group was also significantly lower than that in the UCSEMS group $(3.3 \%$ and $12.5 \%$, respectively; $\mathrm{p}<0.01)$. However, our study differs from previous studies ${ }^{1-4}$ 7-9 11-21 2324 in that we additionally assessed clinical results among patients with extrinsic and intrinsic tumours. No previous studies have compared the clinical results between patients with extrinsic and intrinsic tumours, although assessments have been made either among patients with gastric cancer or those with pancreatic cancer. The incidence of stent ingrowth among patients with pancreatic cancer was $0.0 \%$ with a CSEMS ${ }^{26}$ and $13.8 \%$ $19.7 \%$ with a UCSEMS, ${ }^{27} 28$ while that among patients with gastric cancer was 9.1\% with a CSEMS and 41.2\%-44.4\% with a UCSEMS. ${ }^{8}{ }^{29}$ In the present study, no difference in the proportion of stent ingrowth among patients with extrinsic tumours was found between the CSEMS and UCSEMS groups $(4.2 \%$ and $4.2 \%$, respectively; $p=0.70)$, but the proportion of stent ingrowth among patients with intrinsic tumours was significantly lower in the CSEMS group than the UCSEMS group $(1.6 \%$ and $27.7 \%$, respectively; $\mathrm{p}<0.01)$. This discrepancy can be explained by indirect tumour compression of the SEMS among patients with extrinsic tumours and direct invasion of the SEMS among patients with intrinsic tumours.

CSEMS migration is a challenging problem. The incidence of stent migration in RCTs reportedly ranges from $6.5 \%$ to $32.3 \%$ among patients with a CSEMS and from $0.0 \%$ to $8.3 \%$ in those with a UCSEMS, ${ }^{8} 121316$ and in some studies, the incidence was significantly higher in the CSEMS than the UCSEMS group. ${ }^{812}$ In the present study, the incidence of stent migration was significantly higher in the CSEMS than UCSEMS group $(12.1 \%$ and $2.2 \%$, respectively; $\mathrm{p}<0.01)$. This significant difference remained among patients with extrinsic tumours $(15.3 \%$ and $2.5 \%$, respectively; $\mathrm{p}<0.01)$, but not intrinsic tumours $(6.3 \%$ and $1.5 \%$, respectively; $p=0.35)$. We speculate that extrinsic tumours mainly compress the digestive tract, whereas intrinsic tumours can hold the SEMS in place, even in the presence of a stent cover, because the intrinsic tumours directly invade the digestive tract; thus, stent migration more easily occurs with extrinsic tumours. In previous studies, the incidence of stent migration among patients with pancreatic cancer was $5.3 \%$ in the CSEMS group and $0.0 \%-1.4 \%$ in the UCSEMS group, while the incidence among patients with gastric cancer was $18.2 \%-32.3 \%$ in the CSEMS group and $4.2 \%-8.3 \%$ in the UCSEMS group. ${ }^{8} 29$ These data differ from our results, probably because of the difference in the SEMS. In a single-arm retrospective study using the same flared-ComVi stent, the incidence of stent migration was $23 \%,{ }^{30}$ which is much higher than our result. The novel CSEMS used in our 
study has an anti-migration system at both ends (proximal uncovered flare and distal uncovered area); however, we cannot definitively conclude that this anti-migration system prevents stent migration. Some recent studies have focused on the effectiveness of a CSEMS with other anti-migration systems. Choe et $a^{23}$ reported a SEMS with a 40 -mm-diameter wide funnel shape, and Choi et $a l^{31}$ reported large star-shaped flaps. No distal stent migration occurred with these antimigration systems $(0.0 \%$ for both), although some proximal stent migration occurred $(16.7 \%$ and $11.1 \%$, respectively). Even if a better antimigration system that effectively prevents distal migration is developed, proximal migration still can occur; therefore, stent migration may remain a challenging problem that cannot be prevented.

The incidence of stent overgrowth in RCTs ranged from $0.0 \%$ to $3.2 \%$ in the CSEMS group and from $0.0 \%$ to $3.3 \%$ in the UCSEMS group, ${ }^{8121316}$ with no significant differences between the two groups. In the present study, the incidence of stent overgrowth was significantly higher in the CSEMS group than the UCSEMS group (6.6\% vs $2.2 \%, \mathrm{p}=0.04)$. Although the pathogenesis is unclear, we speculate that stent overgrowth tends to occur in the CSEMS group because the tumour protrudes both ends of the SEMS due to the presence of a stent cover in the CSEMS while the tumour invades between the mesh in the UCSEMS.

In this study, the main primary disease in patients with extrinsic tumours and duodenal stenosis was pancreatic cancer, while that in patients with intrinsic tumours and gastric stenosis was gastric cancer. In the subgroup analysis using the stenotic site (duodenal vs gastric stenosis), we found a significant difference in the proportion of stent migration (CSEMS, $11.1 \%$ vs UCSEMS, $0.0 \% ; \mathrm{p}=0.02$ ) and time to SD $(\log$-rank $\mathrm{p}=0.01)$ in the patients with gastric stenosis. However, we found no significant difference in the proportion of stent migration or time to SD in the intrinsic tumour group in the subgroup analysis using tumour type. The reason for this discrepancy between these subgroup analyses might have been the presence of patients who had pancreatic cancer with gastric stenosis (compression and/or invasion). In these patients, stent migration was prone to occur $(40.0 \%)$ and might have subsequently decreased time to SD. Taking these outcomes into consideration, we speculate that the tumour type may more strongly affect the proportion of stent migration than the stenosis location.

Factors that predict stent-related events have been assessed in previous studies. ${ }^{101720-2332}$ Although predictive factors for clinical failure after GDS placement were mainly assessed, ${ }^{10} 20-2332$ some studies assessed predictive factors for stent patency. Ye et $a l^{17}$ reported that the GOOSS score after GDS placement was a predictive factor, and Jung $e{ }{ }^{18}{ }^{18}$ reported that clinical success was related to stent patency. Furthermore, Yamao et $a l^{21}$ evaluated the factors related not only to clinical failure but also to adverse events, namely, perforation, bleeding and jaundice. In the present study, the predictive factors for SD at 2 months were CSEMS deployment (OR 2.41; 95\% CI 1.34 to $4.33 ; \mathrm{p}<0.01)$ and age (OR $0.98 ; 95 \%$ CI 0.95 to 1.00 ; $\mathrm{p}=0.04)$. The incidences of stent overgrowth and migration among the overall patients were significantly higher in the CSEMS group than the UCSEMS group. We consider that these results affected the multivariable analysis.

In summary, we compared the clinical results between patients with CSEMS and UCSEMS not only among the overall patients but also among patients with extrinsic and intrinsic tumours separately. The proportion of overall SD among the overall patients and patients with extrinsic tumours was significantly higher in the CSEMS group than the UCSEMS group. The time to SD among the overall patients and patients with extrinsic tumours in the CSEMS group was significantly shorter than that in the UCSEMS group. Among patients with intrinsic tumours, however, no difference in the proportion of overall SD or the time to SD was found between the two groups. The proportion of stent migration among patients with extrinsic tumours was significantly higher in the CSEMS group than the UCSEMS group; however, no difference in its proportion among patients with intrinsic tumours was found between the two groups. No difference in the proportion of stent ingrowth among patients with extrinsic tumours was found between the two groups; however, its proportion among patients with intrinsic tumours was significantly lower in the CSEMS group than the UCSEMS group.

This study has two main strengths. First, it was a multicentre prospective randomised study involving a large number of patients. Second, it is the first study of GDSs to compare extrinsic and intrinsic tumours separately. This study also has two main limitations. First, the SEMSs differed: the CSEMSs were $20 \mathrm{~mm}$ in diameter and had a proximal uncovered flare and distal uncovered area, while the UCSEMSs were $22 \mathrm{~mm}$ in diameter. Second, we used the log-rank test to estimate the sample size. The log-rank test is unlikely to detect a difference between groups when the risk of an event is not consistently greater for one group than another. ${ }^{33}$

In conclusion, deployment of a GDS achieved high rates of technical success and clinical success among the overall patients, patients with extrinsic tumours and patients with intrinsic tumours. The proportion of SD among the overall patients and those with extrinsic tumours in the CSEMS group was significantly higher than that in patients in the UCSEMS group. Due to poor patient survival, minor differences between covered and uncovered stents may be less relevant even if statistically significant; however, subgroup analysis would suggest to use covered stents for intrinsic and uncovered stents for extrinsic malignancies.

\section{Author affiliations}

${ }^{1}$ Department of Gastroenterology and Hepatology, Kindai University Faculty of Medicine Hospital, Osakasayama, Osaka, Japan

${ }^{2}$ Second Department of Internal Medicine, Wakayama Medical University, Wakayama, Wakayama, Japan

${ }^{3}$ Clinical Research Center, Kindai University Hospital, Osaka-sayama, Japan

${ }^{4}$ Second Department of Internal Medicine, Osaka Medical College, Takatsuki, Osaka, Japan

${ }^{5}$ Gastroenterology and Hepatology, Osaka Saiseikai Nakatsu Hospital, Osaka, Japan ${ }^{6}$ Innovative Cancer Center, Shimane University Hospital, Matsue, Shimane, Japan

${ }^{7}$ Department of Gastroenterology and Hepatology, Japanese Red Cross Wakayama

Medical Center, Wakayama, Japan

${ }^{8}$ Department of Gastroenterology and Hepatology, Okayama University, Okayama, Japan

${ }^{9}$ Department of Gastroenterology and Hepatology, Kurashiki Central Hospital,

Kurashiki, Japan

${ }^{10}$ Gastroenterology, Bell Land General Hospital, Sakai, Japan

${ }^{11}$ Division of Gastroenterology, Department of Medicine, Kurume University, Kurume, Japan

${ }^{12}$ Division of Gastroenterology, Department of Internal Medicine, Kobe University, Kobe, Hyogo, Japan

${ }^{13}$ Digestive Disease Center, Department of Gastroenterology and Hepatology, Kyoto Katsura Hospital, Kyoto, Japan

${ }^{14}$ Department of Gastroenterology, Matsue Red Cross Hospital, Matsue, Japan

${ }^{15}$ Department of Gastroenterology and Hepatology, JA Hiroshima Koseiren Hiroshima General Hospital, Hatsukaichi, Hiroshima, Japan

${ }^{16}$ Department of Gastroenterology and Hepatology, Toyonaka Municipal Hospital, Toyonaka, Osaka, Japan

${ }^{17}$ Division of Clinical Nutrition, Shiga University of Medical Science, Otsu, Japan 
${ }^{18}$ Digestive Disease Center, The Tazuke Kofukai Medical Research Institute, Kitano Hospital, Osaka, Japan

${ }^{19}$ Department of Gastroenterology, Shikoku Cancer Center, Matuyama, Japan

${ }^{20}$ Department of Gastroenterology, Fukuoka University Chikushi Hospital, Chikushino, Japan

${ }^{21}$ Department of Gastroenterology and Hepatology, Kyoto University, Kyoto, Japan

${ }^{22}$ Department of Gastroenterology and Hepatology, Japanese Red Cross Society Osaka Hospital, Osaka, Osaka, Japan

${ }^{23}$ Division of Medicine and Clinical Science, Tottori University, Yonago, Japan

${ }^{24}$ Department of Gastroenterology, Osaka City General Hospital, Osaka, Japan

${ }^{25}$ Department of Gastroenterology and Hepatology, Kishiwada Tokushukai Hospital, Kishiwada, Japan

${ }^{26}$ Department of Gastroenterology, Shinbeppu Hospital, Beppu, Japan

${ }^{27}$ Gastroenterology and Neurology, Kagawa University, Kagawa, Japan

${ }^{28}$ Department of Gastroenterology, Japanese Red Cross Kyoto Daini Hospital, Kyoto, Japan

${ }^{29}$ Department of Gastroenterology and Hepatology, Tottori Municipal Hospital, Tottori, Japan

${ }^{30}$ Department of Gastroenterology and Hepatology, Tottori Red Cross Hospital, Tottori, Japan

${ }^{31}$ Department of Gastroenterology, Kitaharima Medical Center, Ono, Japan

${ }^{32}$ Department of Gastroenterology, Social Media Corporation Tenyoukai Central Hospital, Kagoshima, Japan

${ }^{33}$ Digestive and Life-style Diseases, Kagoshima University Graduate School of Medicine and Dental Sciences, Kagoshima, Kagoshima, Japan

${ }^{34}$ Department of Gastroenterology, Oita Sanai Medical Center, Oita, Japan

${ }^{35}$ Department of Gastroenterology and Metabolism, Hiroshima University Hospital, Hiroshima, Japan

${ }^{36}$ Department of Gastroenterology, Onomichi General Hospital, Onomichi, Hiroshima, Japan

${ }^{37}$ Department of Internal Medicine, Hiroshima City Hiroshima Citizens Hospital, Hiroshima, Hiroshima, Japan

${ }^{38}$ Department of Gastroenterology, Tenri Hospital, Tenri, Nara, Japan

${ }^{39}$ Department of Gastroenterology, Imakiire General Hospital, Kagoshima, Japan

${ }^{40}$ Department of Gastroenterology, Hyogo Prefectural Awaji Medical Center, Sumoto, Japan

${ }^{41}$ Third department of Internal Medicine, Nara Medical University, Kashihara, Nara, Japan

${ }^{42}$ General Medicine 2, Kawasaki Medical School, Okayama, Japan

Contributors $\mathrm{KY}$ : contributed to the study design, recruited patients to the study, treated patients, contributed to the running of the study, analysed the results and wrote the manuscript draft. MK : designated the study, recruited patients to the study, treated patients, contributed to the running of the study, reviewed the analysis, and reviewed and contributed to the writing of the manuscript. $Y C$ : contributed to the study design, analysed the results, and reviewed and contributed to the writing of the manuscript. SB : contributed to the study design, recruited patients to the study, treated patients, contributed to the running of the study, reviewed the analysis, and reviewed and contributed to the writing of the manuscript. SH : contributed to the study design, recruited patients to the study, treated patients, contributed to the running of the study and reviewed the analysis. All other authors contributed to the study design, recruited patients to the study, treated patients and contributed to the running of the study.

Funding The authors have not declared a specific grant for this research from any funding agency in the public, commercial or not-for-profit sectors.

Competing interests None declared.

Patient consent for publication Not required.

Ethics approval The study was approved by the ethical committees at 41 tertiary centres in Japan.

Provenance and peer review Not commissioned; externally peer reviewed.

Data availability statement Data are available upon reasonable request. We are ready to respond to any inquiries about the detailed data in this study.

Supplemental material This content has been supplied by the author(s). It has not been vetted by BMJ Publishing Group Limited (BMJ) and may not have been peer-reviewed. Any opinions or recommendations discussed are solely those of the author(s) and are not endorsed by BMJ. BMJ disclaims all liability and responsibility arising from any reliance placed on the content. Where the content includes any translated material, BMJ does not warrant the accuracy and reliability of the translations (including but not limited to local regulations, clinical guidelines, terminology, drug names and drug dosages), and is not responsible for any error and/or omissions arising from translation and adaptation or otherwise.

Open access This is an open access article distributed in accordance with the Creative Commons Attribution Non Commercial (CC BY-NC 4.0) license, which permits others to distribute, remix, adapt, build upon this work non-commercially, and license their derivative works on different terms, provided the original work is properly cited, appropriate credit is given, any changes made indicated, and the use is non-commercial. See: http://creativecommons.org/licenses/by-nc/4.0/.

\section{ORCID iDs}

Masayuki Kitano http://orcid.org/0000-0001-6885-9223

Takeshi Ogura http://orcid.org/0000-0003-2916-6568

Shigeki Bamba http://orcid.org/0000-0002-4108-5894

\section{REFERENCES}

1 Mehta S, Hindmarsh A, Cheong E, et al. Prospective randomized trial of laparoscopic gastrojejunostomy versus duodenal stenting for malignant gastric outflow obstruction. Surg Endosc 2006;20:239-42.

2 Chandrasegaram MD, Eslick GD, Mansfield CO, et al. Endoscopic stenting versus operative gastrojejunostomy for malignant gastric outlet obstruction. Surg Endosc 2012;26:323-9.

3 Jeurnink SM, Steyerberg EW, Hof G van 't, Hof G, et al. Gastrojejunostomy versus stent placement in patients with malignant gastric outlet obstruction: a comparison in 95 patients. J Surg Oncol 2007;96:389-96.

4 Jeurnink SM, Steyerberg EW, van Hooft JE, et al. Surgical gastrojejunostomy or endoscopic stent placement for the palliation of malignant gastric outlet obstruction (SUSTENT study): a multicenter randomized trial. Gastrointest Endosc 2010;71:490-9.

5 Hamada T, Hakuta R, Takahara N, et al. Covered versus uncovered metal stents for malignant gastric outlet obstruction: systematic review and meta-analysis. Dig Endosc 2017;29:259-71.

6 Adler DG, Baron TH. Endoscopic palliation of malignant gastric outlet obstruction using self-expanding metal stents: experience in 36 patients. Am J Gastroenterol 2002;97:72-8

7 Lee KM, Choi SJ, Shin SJ, et al. Palliative treatment of malignant gastroduodenal obstruction with metallic stent: prospective comparison of covered and uncovered stents. Scand J Gastroenterol 2009;44:846-52

$8 \mathrm{Kim} \mathrm{CG}$, Choi IJ, Lee JY, et al. Covered versus uncovered self-expandable metallic stents for palliation of malignant pyloric obstruction in gastric cancer patients: a randomized, prospective study. Gastrointest Endosc 2010;72:25-32.

9 Isayama H, Sasaki T, Nakai Y, et al. Management of malignant gastric outlet obstruction with a modified triple-layer covered metal stent. Gastrointest Endosc 2012;75:757-63.

10 Sasaki T, Isayama $H$, Nakai $Y$, et al. Predictive factors of solid food intake in patients with malignant gastric outlet obstruction receiving self-expandable metallic stents for palliation. Dig Endosc 2012;24:226-30.

11 Sasaki T, Isayama H, Maetani I, et al. Japanese multicenter estimation of WallFlex duodenal stent for unresectable malignant gastric outlet obstruction. Dig Endosc 2013:25:1-6.

12 Lim SG, Kim JH, Lee KM, et al. Conformable covered versus uncovered selfexpandable metallic stents for palliation of malignant gastroduodenal obstruction: a randomized prospective study. Dig Liver Dis 2014;46:603-8.

13 Maetani I, Mizumoto Y, Shigoka H, et al. Placement of a triple-layered covered versus uncovered metallic stent for palliation of malignant gastric outlet obstruction: a multicenter randomized trial. Dig Endosc 2014;26:192-9.

14 Tringali A, Didden P, Repici A, et al. Endoscopic treatment of malignant gastric and duodenal strictures: a prospective, multicenter study. Gastrointest Endosc 2014;79:66-75.

$15 \mathrm{Kim} J \mathrm{~W}$, Jeong JB, Lee KL, et al. Comparison between uncovered and covered self-expandable metal stent placement in malignant duodenal obstruction. World J Gastroenterol 2015;21:1580-7.

16 Lee $\mathrm{H}, \mathrm{Min} \mathrm{B}-\mathrm{H}$, Lee $\mathrm{JH}$, et al. Covered metallic stents with an anti-migration design vs. uncovered stents for the palliation of malignant gastric outlet obstruction: a multicenter, randomized trial. Am J Gastroenterol 2015;110:1440-9.

17 Ye B-W, Lee K-C, Hsieh Y-C, et al. Self-Expandable metallic stent placement in malignant gastric outlet obstruction: a comparison between 2 brands of stents. Medicine 2015;94:e1208.

18 Jung $\mathrm{K}$, Ahn JY, Jung $\mathrm{H}$-Y, et al. Outcomes of endoscopically inserted self-expandable metal stents in malignancy according to the type of stent and the site of obstruction. Surg Endosc 2016;30:4001-10.

19 Park J-H, Lee JH, Song H-Y, et al. Over-the-wire versus through-the-scope stents for the palliation of malignant gastric outlet obstruction: a retrospective comparison study. Eur Radiol 2016;26:4249-58.

20 Shin YS, Choi CW, Kang DH, et al. Factors associated with clinical failure of self-expandable metal stent for malignant gastroduodenal obstruction. Scand $J$ Gastroenterol 2016;51:103-10.

21 Yamao K, Kitano M, Kayahara T, et al. Factors predicting through-the-scope gastroduodenal stenting outcomes in patients with gastric outlet obstruction: a large multicenter retrospective study in West Japan. Gastrointest Endosc 2016:84:757-63.

22 Hori Y, Naitoh I, Hayashi K, et al. Predictors of outcomes in patients undergoing covered and uncovered self-expandable metal stent placement for malignant gastric outlet obstruction: a multicenter study. Gastrointest Endosc 2017;85:340-8. 
23 Choe JW, Hyun JJ, Lee D-W, et al. Comparison on the efficacy between partially covered self-expandable metal stent with funnel-shaped enlarged head versus uncovered self-expandable metal stent for palliation of gastric outlet obstruction. Gastroenterol Res Pract 2018;2018:1-8.

24 Jeong JY, Han JK, Kim AY, et al. Fluoroscopically guided placement of a covered self-expandable metallic stent for malignant antroduodenal obstructions: preliminary results in 18 patients. AJR Am J Roentgenol 2002; 178:847-52.

25 Pan Y-min, Pan J, Guo L-kun, et al. Covered versus uncovered self-expandable metallic stents for palliation of malignant gastric outlet obstruction: a systematic review and meta-analysis. BMC Gastroenterol 2014;14:170.

26 Tsauo J, Yoo M-W, Song H-Y, et al. Partially-covered stent placement versus surgical gastrojejunostomy for the palliation of malignant gastroduodenal obstruction secondary to pancreatic cancer. Abdom Radiol 2016;41:2233-40.

27 Kobayashi S, Ueno M, Kameda R, et al. Duodenal stenting followed by systemic chemotherapy for patients with pancreatic cancer and gastric outlet obstruction. Pancreatology 2016;16:1085-91.
28 Chang K-B, Ye B-W, Chou C-K, et al. Outcomes of enteral metallic stent in patients with pancreatic carcinoma and gastric outlet obstruction: a single center experience. $J$ Formos Med Assoc 2020;119:238-246.

29 Cho YK, Kim SW, Hur WH, et al. Clinical outcomes of self-expandable metal stent and prognostic factors for stent patency in gastric outlet obstruction caused by gastric cancer. Dig Dis Sci 2010;55:668-74.

30 Takahara N, Isayama H, Nakai Y, et al. A novel partially covered self-expandable metallic stent with proximal flare in patients with malignant gastric outlet obstruction. Gut Liver 2017;11:481-8.

31 Choi YK, Ahn JY, Na HK, et al. Winged partially covered self-expandable metal stent to prevent distal migration in malignant gastric outlet obstruction. Dig Dis Sci 2018;63:3409-16.

32 Sato T, Hara K, Mizuno N, et al. Type of combined endoscopic biliary and gastroduodenal stenting is significant for biliary route maintenance. Intern Med 2016;55:2153-61.

33 Bland JM, Altman DG. The logrank test. BMJ 2004;328:1073. 